Thorax (1950), 5, 194.

\title{
A MODIFIED CONCEPTION OF PHRENIC NERVE CRUSH AND PNEUMOPERITONEUM THERAPY
}

\author{
BY \\ WALLACE FOX \\ From Preston Hall Hospital, Aylesford, Kent
}

The object of this paper is to present a modified view of the mode of action of phrenic nerve crush and pneumoperitoneum therapy in pulmonary tuberculosis.

In a study of mediastinal shift in a series of 80 cases of phrenic nerve crush and pneumoperitoneum therapy (Fox, 1950) it was found that the average mediastinal shift to the left in the cases with right phrenic paralysis was $1.6 \mathrm{~cm}$., and to the right in those with left phrenic paralysis was $1.5 \mathrm{~cm}$. Furthermore, of these 80 cases, only 10 had no demonstrable mediastinal shift.

It is therefore apparent that mediastinal shift is present in the large majority of cases following phrenic nerve crush and pneumoperitoneum therapy, and the figures quoted demonstrate that the generally accepted view of the form of the collapse produced by phrenic nerve crush and pneumoperitoneum therapy is fallacious. The literature on phrenic nerve crush and pneumoperitoneum therapy in pulmonary tuberculosis gives the impression that the sole spatial effect of the treatment is to reduce the apico-basal diameters of the lungs. The two hemidiaphragms are regarded as plungers situated at the thoracic outlet. These plungers become elevated as a result of the therapy, and so there is a diminution in the apico-basal diameters, the greater reduction occurring on the side of the paralysed hemidiaphragm. In this view each hemithorax can be represented as a right-angled triangle the base of which moves upwards as a result of the therapy, thus producing a smaller rightangled triangle (Fig. 1). This, however, is inaccurate, for not only are the bases moving, but in addition the perpendicular sides move too, so that an acute angle is produced on the paralysed side and an obtuse angle on the unparalysed side in any case where the mediastinum is not markedly rigid (Fig. 2). It is important to appreciate this point because it materially alters the mechanism of the therapy on both sides. Each side will now be considered in turn.

The Paralysed Side.-Too much attention has been focused on the reduction of the apico-basal diameter of the lung, even though this is often very considerable. There is, in addition, an increase in the dimensions of the lung from side to side and from before back, the mechanism and significance of which deserves careful study.

The broadening from side to side is most marked at the basal portion of the lung field. It is, however, by no means always confined to the lung base, as is evidenced by the tracheal shift towards the unparalysed side which is present in some cases. 
This broadening of the lung field is due to two factors. These are, first, the mediastinal shift away from the paralysed side, and second, the hemithorax as a whole is broadened due to the increased upward and outward movements of the ribs which result from the phrenic paralysis (Fig. 3). The second factor deserves further consideration as its occurrence has been the source of some divergence of opinion. Thus Felix (1925) found that diaphragmatic paralysis was followed by diminished lateral expansion of the lower ribs, whereas Lemon (1928) was unable to find any alteration of costal movement following diaphragmatic paralysis. There is, however, a considerable body of opinion in favour of the view that increased movement of the homolateral ribs does in fact follow diaphragmatic paralysis. The first report of increased homolateral rib movement was by Hoover (1913) who studied it both clinically and experimentally. Roith (1926) reported an increase in movement of all the homolateral ribs following hemidiaphragmatic paralysis in man. He advised section of the upper intercostal nerves to counteract this in cases where the diaphragm was paralysed in the treatment of apical pulmonary tuberculosis. Schnippenkötter (1926) in an experimental study in cats found increased homolateral excursion of the ribs with hypertrophy of the intercostal
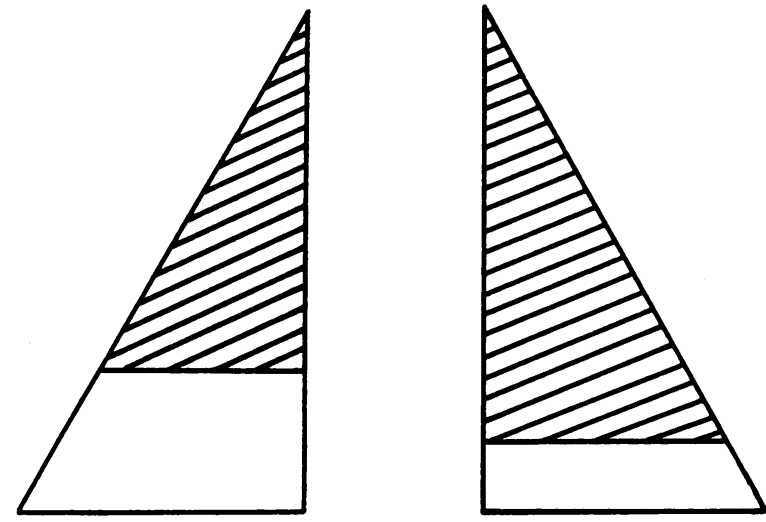

FIG. 1.-Diagram to illustrate the generally accepted mode of collapse in a right phrenic nerve crush and pneumoperitoneum. The lined areas represent the lungs after the establishment of the therapy.
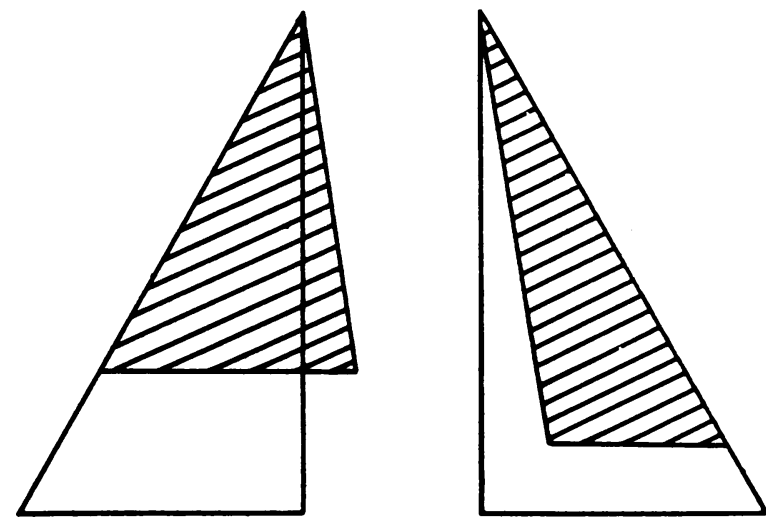

FIG. 2.-Diagram to illustrate the modified view of the collapse, showing the effect of the mediastinal shift.
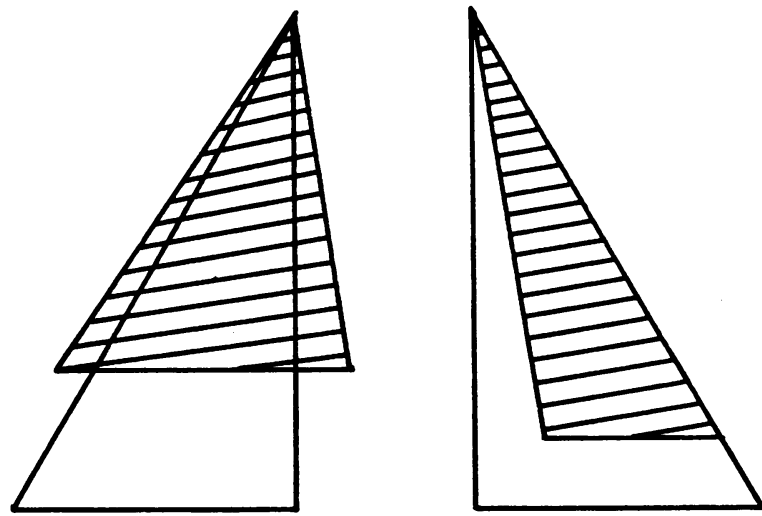

FIG. 3.-Diagram to illustrate the modified view of the collapse with both the mediastinal shift and the altered shape of the right hemithorax represented. 
musculature. Head (1930) in a study of 200 patients after phrenico-exeresis concluded that "in the majority there is an increased outward and upward movement of the lower ribs on the affected side and always, unless pulmonary disease has greatly contracted the upper thorax, an increased movement of the upper ribs. Thus, costal breathing is increased throughout the affected side." He confirmed these observations in an experimental study in dogs. Sisti and Soricelli (1936) in an $x$-ray kymographic study after phrenic nerve crush in 16 patients found increased homolateral rib movement. Gale and Middleton (1932) performed a crucial series of experiments in human volunteers. They made direct kymographic recordings of the movements of the third and eighth ribs on both sides in several patients both before and after a phrenic nerve paralysis, and they found that an increase in movement of these two ribs on the homolateral side resulted from the paralysis of the diaphragm. It is also of interest that in one patient with a very diseased lung and mediastinal distortion, the paralysis resulted in increased movements of the ribs on both sides. This, they considered, was due to increased mobility of the mediastinum and the extent of the disease requiring compensatory increased respiration in the opposite lung. Pinner (1937) reported that he had confirmed Head's (1913) observation that there was an increased lateral flare of the lower ribs on the side of the paralysed diaphragm. Reichert (1933) in an experimental study in dogs found no alteration in the shape of the chest after unilateral diaphragmatic paralysis, but following bilateral paralysis observed a definite enlargement of the thoracic cage.

The presence of a pneumoperitoneum in addition to the phrenic nerve crush often makes this compensatory movement of the ribs most obvious, for I have observed it quite frequently at radioscopy in patients with phrenic crush and pneumoperitoneum therapy. Its presence, however, cannot invariably be detected on radioscopy. In particular it is usually not apparent in patients with highly fibrotic lesions which normally diminish the respiratory excursion of the homolateral ribs. Further, a series of radiological observations analogous to those of Reichert (1933) in dogs were made. In the series of 80 patients in which the mediastinal shift was studied, when comparable radiographs of the chest before treatment was begun and after the therapy was fully established were superimposed, the chest was found to be broadened in many cases by at least a centimetre and in some cases by over two centimetres. In no case in the entire series was there diminution in the diameter of the chest. These observations also suggest that a broadening of the chest occurs after phrenic paralysis and pneumoperitoneum. The increase in the anteroposterior dimension of the lung follows automatically from the increased upward and outward movement of the ribs (Head, 1930).

Another factor relating to the effect of the therapy on the antero-posterior and side-to-side dimensions of the lung must be considered, for Hanrahan (1949) suggests that concentric relaxation will be produced since the thorax is cone-shaped and lung tissue may ascend to a place where the area of cross section is considerably reduced. This view, however, does not take into consideration the increased dimensions of the hemithorax or the mediastinal shift away from the side of the diaphragmatic paralysis. Moreover, the chest is only very approximately cone-shaped and a very considerable elevation of the diaphragm must occur before any wedging effect can be expected. 
From a dynamic point of view the diaphragmatic pumping action is considerably reduced or even completely abolished, but even this is in part offset by the increased movement of the homolateral ribs. Hence the vertical reduction of the lung field following phrenic nerve crush and pneumoperitoneum therapy is the only measure of collapse which may be expected to be of therapeutic significance, and this is in part offset by the increased dimensions of the lung in the transverse and anteroposterior planes. Since the antero-posterior and lateral dimensions of the lung are not reduced there can be no concentric relaxation of the lung on the paralysed side, the optimal condition for healing in an artificial pneumothorax. Mechanically, this is an obvious limiting factor which may well explain the failure to control the disease and close cavities in some cases, even when the reduction of the apico-basal diameter is very considerable.

The Unparalysed Side.-The same mechanical factors that have been considered on the paralysed side play their part in the mechanism of the therapy on the unparalysed side, but their relative importance is altered. Thus, there is a reduction in the apico-basal diameter of the lung, but this is considerably less than on the paralysed side. The mediastinal shift towards the unparalysed side produces a reduction of the transverse diameter of the lung, and its effect is comparable to that produced by a contralateral artificial pneumothorax. There is then on this side a reduction of both the apico-basal and side-to-side dimensions of the lung so that there is a nearer approach to the theoretically desirable concentric relaxation of the lung than occurs on the paralysed side. A reduction of the antero-posterior diameter of the lung cannot be expected, however, and there is never a sufficiently high elevation of the diaphragm to allow the wedging effect described by Hanrahan (1949) to occur on this side.

It is possible that the pneumoperitoneum alone produces increased rib movements due to the elevation of the hemidiaphragm. If this is so, however, my radioscopic observations suggest that the degree of increased movement in many cases at any rate is less than on the paralysed side, for, as already stated, the ribs on the paralysed side can usually be seen to move more. Again, the possibility that increased rib movement on this side may sometimes result from the phrenic paralysis alone must also be considered, since the direct kymographic observations in one of Gale and Middleton's (1932) cases showed that this could occur. The difficulty of making observations on this factor is due to the absence of a standard with which to compare the rib movement on this side.

Although the pumping action of the diaphragm still continues there is a reduction in the range of its excursion (Mitchell, Hiatt, McCain, Easom, and Thomas, 1947), so that the diaphragmatic dynamic factor is diminished.

The effect of the therapy both statically and dynamically, therefore, is different on the two sides and it is the purpose of this paper to emphasize this point. It is of course not intended to throw doubt on the generally accepted view that a greater therapeutic effect can be expected on the paralysed side than on the unparalysed side in cases with a phrenic nerve crush and pneumoperitoneum. Figs. 4 and 5, produced by superimposing tracings of comparable radiographs taken before treatment was begun and after the full establishment of the phrenic nerve crush and pneumo- 


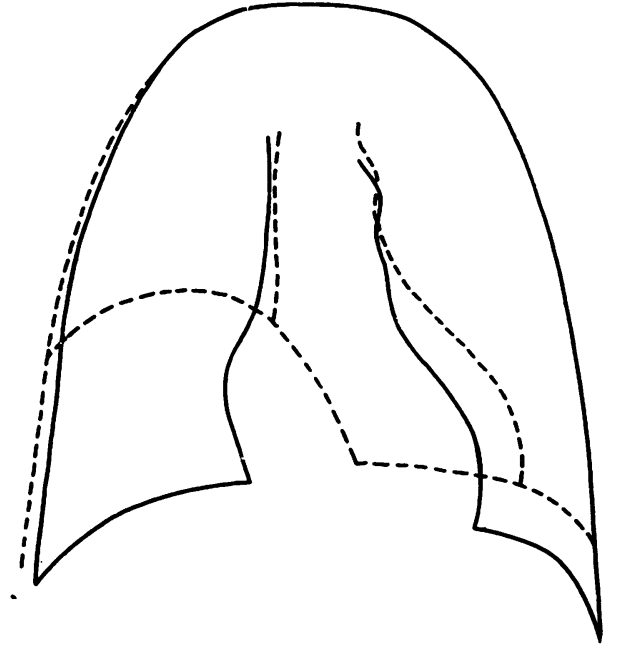

Fig. 4.

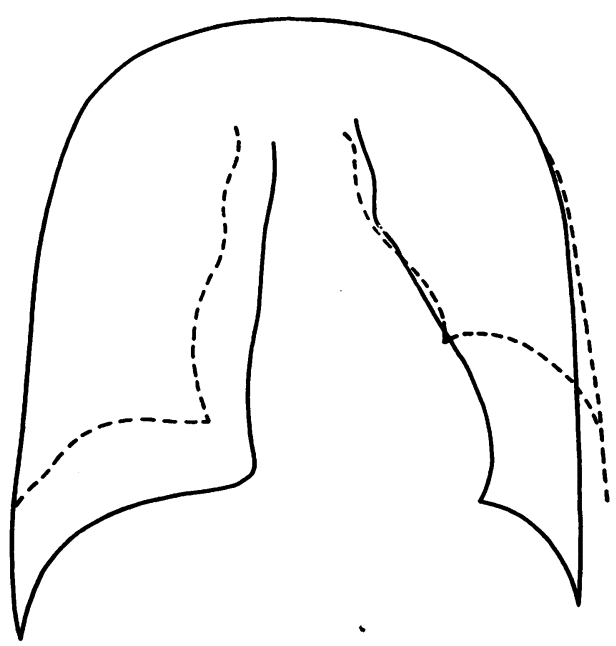

Fig. 5.

FIGs. 4 and 5.-Superimposed tracings of the outlines of the chest and mediastinum before the beginning of phrenic nerve crush and pneumoperitoneum therapy and after its full establishment. The mediastinal shift and reduction of the lung volume on the unparalysed side is demonstrated. Fig. 4 represents a right phrenic nerve crush and pneumoperitoneum, and Fig. 5 a left phrenic nerve crush and pneumoperitoneum. Typical rather than extreme examples have been selected in order to demonstrate the usual degree of modification.

peritoneum, demonstrate the mediastinal shift and the considerable reduction of lung volume produced on the unparalysed side in the presence of a mobile mediastinum.

One of the striking features of phrenic nerve crush and pneumoperitoneum therapy is the unpredictability of the results in any individual case. The results of the therapy depend on the character of the intrapulmonary lesion; that is, its site, its retractability, fibrosis, and the size of cavities, the nature of their walls and draining bronchi, and the presence of pleural obliteration. It will also depend on the mechanical factors of the therapy ; namely, the alterations of dimensions of the lung in all three planes and the modifications of both the diaphragmatic and rib movements produced by the therapy. It is therefore not surprising that the results are often unpredictable.

\section{SUMMARY}

The movement of the mediastinum away from the side of the paralysed hemidiaphragm in phrenic nerve crush and pneumoperitoneum therapy is an important mechanical feature. It results in an increase in the side-to-side dimensions of the lung on the paralysed side and a decrease on the unparalysed side. There is moreover a compensatory rib movement on the paralysed side. These factors necessitate a modified conception of the mode of collapse produced by the therapy.

I am indebted to Professor F. R. G. Heaf for his helpful criticisms of this paper. 


\section{REFERENCES}

Felix, W. (1925). Ergebn. Chir. Orthop., 18, 690.

Fox, W. (1950). Thorax, 5, 183.

Gale, J. W., and Middleton, W. S. (1932). Amer. Rev. Tuberc., 25, 99.

Hanrahan, M. (1949). Irish J. med. Sci., 6S., 112.

Head, J. R. (1930). Surg. Gynec. Obstet., 50, 929.

Hoover, C. F. (1913). Arch. intern. Med., 12, 214

Lemon, W. S. (1928). Arch. Surg., Chicago, 17, 840.

Mitchell, R. S., Hiatt, J. S., McCain, P. P., Easom, H. F., and Thomas, C. D. (1947). Amer. Rev. Tuberc., 55, 307.

Pinner, M. (1937). In Alexander's Collapse Therapy of Pulmonary Tuberculosis. Charles C. Thomas \& Co. Springfield, Illinois. P. 50.

Roith, O. (1926). Mitt. Grenzgeb. Med. Chir., 39, 377.

Reichert, F. L. (1933). J. thorac. Surg., 2, 349.

Schnippenkötter, W. (1926). Beitr. klin. Tuberc., 65, 56.

Sisti, M. A., and Soricelli, F. (1936). Arch. Radiol., 12, 108. Quoted from Audrey G. Morgan (1937). Amer. J. Roentgenol., 38, 802. 